\title{
Was ist nun das Blutdruckziel bei Diabetikern?
}

\author{
Der Streit um die Blutdruckzielwerte ist nach wie vor in vollem Gange, wie wir im Seminar ab S. 85 \\ darstellen. Zumindest bei Diabetikern könnte nun eine neue, sehr große Metaanalyse Klarheit schaffen.
}

_ Für eine umfangreiche Metaanalyse
zu Blutdruckzielwerten wurden Studien
mit einer Dauer von mindestens $12 \mathrm{Mo-}$
naten und mindestens 100 Patienten mit
Diabetes (überwiegend Typ 2) selektiert.
Die Arbeiten mussten ein Antihyperten-
sivum mit Placebo oder Kombinations-
mit Monotherapie vergleichen sowie be-
stimmte Zielwerte anstreben.

49 Studien mit 73.738 Patienten erfüllten die Kriterien. Die Studiendauer betrug im Schnitt 3,7 Jahre, die Blutdruckdifferenz zwischen Kontroll- und Interventionsgruppe 10,2 $\mathrm{mmHg}$. Die Auswertung ergab, dass der Erfolg der antihypertensiven Therapie entscheidend vom Ausgangswert abhängt:

- Bei Werten über $150 \mathrm{mmHg}$ kommt es bei einer mittleren Senkung um 10 mmHg zu signifikanten Abnahmen von Gesamt- und kardiovaskulärer Mortalität, Herzinfarkten, Schlaganfällen und Herzinsuffizienz.

- Schon zwischen 140 und 150 mmHg wird der Nutzen bei allen Endpunkten geringer. Nur bei Gesamtmortalität, Herzinfarkt und Herzinsuffizienz bleibt er signifikant.

- Bei Ausgangswerten unter 140 mmHg verringert sich der Benefit der Therapie weiter. Kardiovaskuläre und Gesamtmortalität steigen sogar signifikant an.

Bei Betrachtung der unter Therapie erreichten Werte gilt: Werte von 130-140 mmHg senken das Risiko nicht stärker als Werte über $140 \mathrm{mmHg}$. Ein Blutdruck unter $130 \mathrm{mmHg}$ ist tendenziell schlechter - ausgenommen zur Verhinderung von Schlaganfällen.
- Brunström M, Carlberg B. Effect of antihypertensive treatment at different blood pressure levels in patients with diabetes mellitus: systematic review and meta-analyses. BMJ. 2016;352:i717

\section{KOMMENTAR}

Sowohl für als auch gegen strenge Blutdruckzielwerte sprechen jeweils eine Vielzahl von Studien und Metaanalysen. Doch womöglich ist die aggressive Therapie selbst eine entscheidende Variable. Kürzlich führte sie in einer englischen Studie von niedergelassenen Ärzten zu unerwarteten Nachteilen: Patienten kamen seltener zu Kontrollen, nahmen weniger zuverlässig die Medikamente, machten die Dosissteigerung für Befindlichkeitsstörungen verantwortlich und lehnten weitere Verordnungen ab. Da sticht der graue Alltag jede Evidenz aus Studien.

Prof. Dr. med. H. Holzgreve

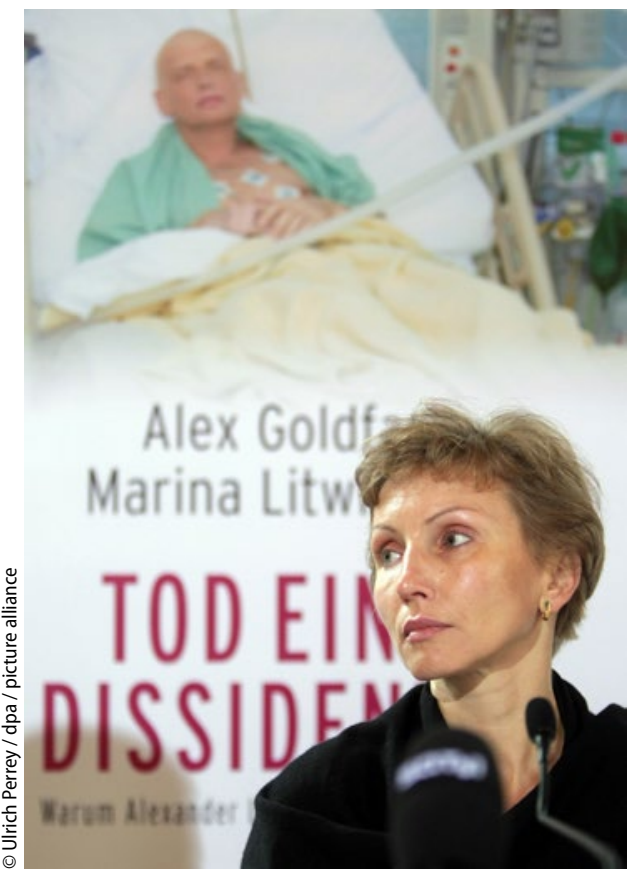

Alexander Litwinenkos Ehefrau Marina kämpft seit seinem Tod für eine vollständige Aufklärung des Falls.

\section{Ein fast perfekter Mord an einem Staatsfeind}

Nach Abschluss der behördlichen Untersuchungen dürfen die Londoner Ärzte von Alexander Litwinenko erstmals über die Behandlung im Jahr 2006 berichten. Der Fall klingt wie eine James-Bond-Geschichte. Alle Symptome sprachen zunächst für eine banale Gastroenteritis. Doch als es dem Patienten nach einer Woche immer schlechter ging, offenbarte er seine Identität: Er war ein ehemaliger Offizier des sowjetischen Geheimdienstes, der zu den Briten übergelaufen war. Nun befürchtete er, Opfer einer Vergiftung zu sein.

In den folgenden zwei Wochen kam es zu Neutro- und Thrombozytopenie, Alopezie und Mukositis. Die Ärzte suchten fieberhaft nach einer Ursache. Leicht erhöhte Werte unterhalb der toxischen Grenze weckten den Verdacht auf eine Thalliumvergiftung, doch dies stellte sich als falsch heraus. Auf die richtige Spur kam man erst einen Tag vor Litwinenkos Tod infolge von Multiorganversagen: Er war mit Polonium-210 vergiftet worden. Die tödlichen Dosen der radioaktiven Substanz wurden postmortal in mehreren Organen einwandfrei verifiziert.

Die Herstellung von Polonium-210 ist äußerst schwierig, bedarf entsprechender Spezialisten und Ressourcen. Das Produkt ist ein farbloses, wasserlösliches Salz, das im Körper schnell resorbiert wird. Vermutlich haben es zwei ehemalige KGB-Agenten in Litwinenkos Tee gemischt. Die Alphastrahlung des inkorporierten Radionuklids kann man mit dem üblichen Geigerzähler nicht nachweisen.

Die Ärzte betonen, dass sie ihren Patienten auch bei einer früheren Diagnose nicht hätten retten können, da die Polonium-210-Dosis zu hoch war. Sie hatte sich binnen Stunden im gesamten Körper verteilt - zu dem Zeitpunkt war Litwenenko bereits todgeweiht.

Prof. Dr. med. H. Holzgreve

- Nathwani AC, Down JF, Goldstone J et al. Polonium-210 poisoning: a first-hand account. Lancet. 2016;388:1075-80 